\title{
Determining the Adulteration of Diesel by an Optical Method
}

\author{
Anwar Sadat \\ Electronics Engineering Department \\ Aligarh Muslim University, Aligarh, UP., India
}

\begin{abstract}
Diesel is one of the fuels used in automobiles. But diesel is often adulterated with Kerosene which harms the engine and reduces the life of automobiles. An electronic technique has been developed to determine the adulteration in diesel by kerosene, in which the light is passed in an optical fiber, the cladding of which is removed over a small area, where the evanescent wave interacts with the measurand. The light received is converted into a proportional current and then into voltage. The technique is simple, compact and portable. The results obtained are similar to the results obtained from UVVIS spectrophotometer.
\end{abstract}

\section{Keywords}

Adulteration, kerosene, diesel

\section{INTRODUCTION}

Most commonly used commercial automotive fuel available on the Indian subcontinent is diesel. They are generally adulterated with kerosene, as the price of kerosene, distributed through public distribution system is kept low due to social and economical considerations. Kerosene being the most popular adulterants for diesel is much similar in chemical structure to diesel and thus mixes with almost no aberration in the properties of automotive fuel [1-2].

Adulterated fuels make exhaust gases more poisonous, worsening the pollution crisis and causing acute respiratory infections and other ailments. Adulteration of diesel with kerosene decreases diesel's lubricating function, leading to faster wear and tear of the pistons and thus requires higher maintenance costs. In addition, the soot particles carried by diesel exhausts also have unburnt and harmful hydrocarbons from the kerosene [3]

Hence it becomes necessary to check their adulteration as it may affect the performance and may damage the appliances in the worst possible case.

A number of methods are available for measuring adulteration of diesel, by kerosene viz; the filter test, testing through specific gravity, viscosity, odor based method [4], ultrasonic technique [5], titration technique [6-8] etc. The specific gravity measurement suffers significant inaccuracy in the measurement, whereas the viscosity measurement method has the drawback of generating the same viscosity of the adulterated sample by adding a small amount of high viscosity lubricating oils.

Fiber optic sensors offer several advantages compared to conventional non-optical sensors. It provides the possibility of sensor technology that is lightweight, small size, potentially multiplexable, immune to electromagnetic interference (EMI), without electromagnetic susceptibility (EMS), and at the same time, it does not require electrical current at the sensing point [9].
In the optical fiber, the light is guided by the core, but a small percentage of the field travels in the cladding. The cladding is removed, the evanescent wave interacts with the measurand providing the basis for sensing. For the fluids obeying the Lambert-Beer law of absorption, the evanescent absorbance depends linearly on both exposed fiber length and the fluid concentration [10-11]. This paper deals with an electronic technique to determine the adulteration of diesel caused by kerosene. The method described in this paper is simple, compact and portable as compared to the methods already available to check the adulteration.

\section{EXPERIMENTAL METHOD}

An LED is placed at one end of the fiber provides sufficient radiant energy over the wavelength region where absorption is to be measured. The light is guided inside an optical fiber through the principle of total internal reflection. The cladding of an optical fiber is removed over a small length of the fiber. Thus, evanescent wave is able to interact with the measurand, providing the basis for sensing. The light received at the other end of the fiber is converted into a proportional current using a photodiode. This current is further converted into a proportionate output voltage using a current to voltage converter. The resulting voltage can be measured using a sensitive digital voltmeter.

The MFOE 71, 9508, Mexico, LED was used which provide the radiation at the wavelength of $630 \mathrm{~nm}$. The length of the optical fiber used was $30 \mathrm{~cm}$ with a core diameter of $1 \mathrm{~mm}$, from which cladding of $6 \mathrm{~cm}$ was removed from the central region. The material of the core is polymethylmetacrilate (PMMA), with a refractive index of 1.492 . The cladding is made of, fluorinated polymers with refraction index of 1.417. The photo detector used is MFOD71, 9432, Mexico which converts the light intensity into the proportionate current.

The bulk absorbance of the diesel at different adulteration level was measured with ELICO SL 164 Double beam UVVIS spectrophotometer at $630 \mathrm{~nm}$ wavelength.

\section{EXPERIMENTAL SETUP}

The experimental set-up of the proposed electronic technique to determine the adulteration is shown in Fig. 1. It consists of $5 \mathrm{~V}$ constant dc supply connected to the LED. The light emitted by an LED is proportional to the forward current. Light emitting diodes are designed to produce coherent light at $630 \mathrm{~nm}$ wavelength with a very narrow bandwidth.

Silicon photodiode is being used as the photo detector in the circuit. The current of the photo detector is proportional to the intensity of the incident light falling on it. The photo detector is in the photovoltaic mode, which offers lower noise and therefore, is better suited for measurement and instrumentation applications. The Op Amp's input impedance being very high, the photo diode current flows through the 
feedback resistor $R$. This current is converted into a

$$
V_{0}=I_{L} \times R
$$

proportional voltage given by:

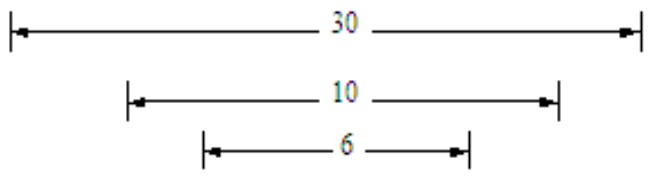

$100 \mathrm{kohm}$

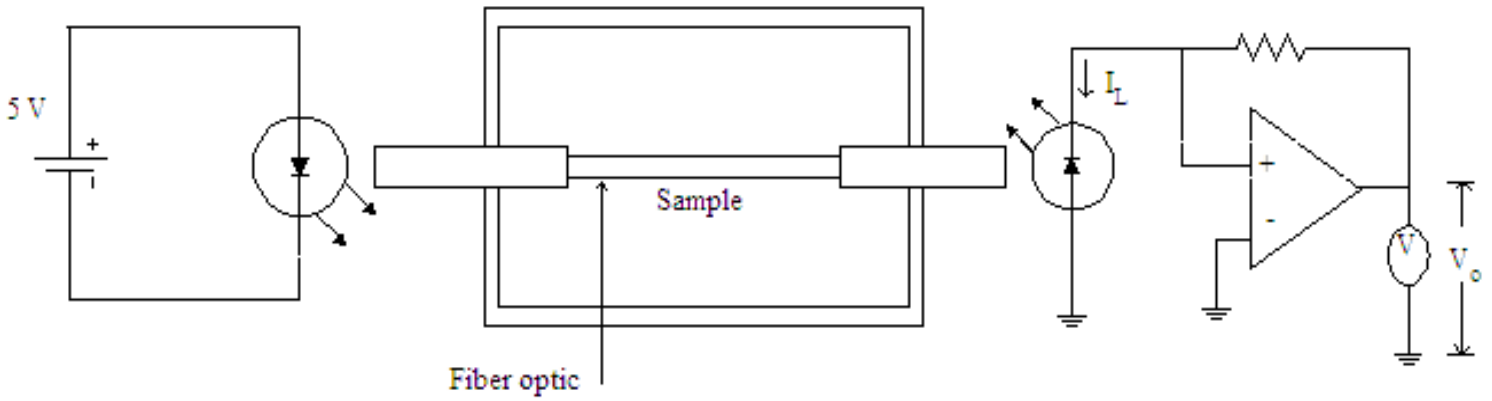

Figure 1. Experimental setup

\section{EXPERIMENTAL RESULTS}

The bulk absorbance of diesel at different adulteration levels is measured at $630 \mathrm{~nm}$ of wavelength. The absorbance measured is with relative to pure diesel. The variation of bulk absorbance with increase in the percentage of kerosene in diesel is shown in Fig. 2. It can be observed that the absorbance increases with increase in kerosene adulteration.
The variation of the output voltage for each value of the concentration of kerosene in diesel is measured with the proposed electronic technique is shown in Fig. 3. The output voltage decreases almost linearly with increase in the percentage of kerosene in diesel.

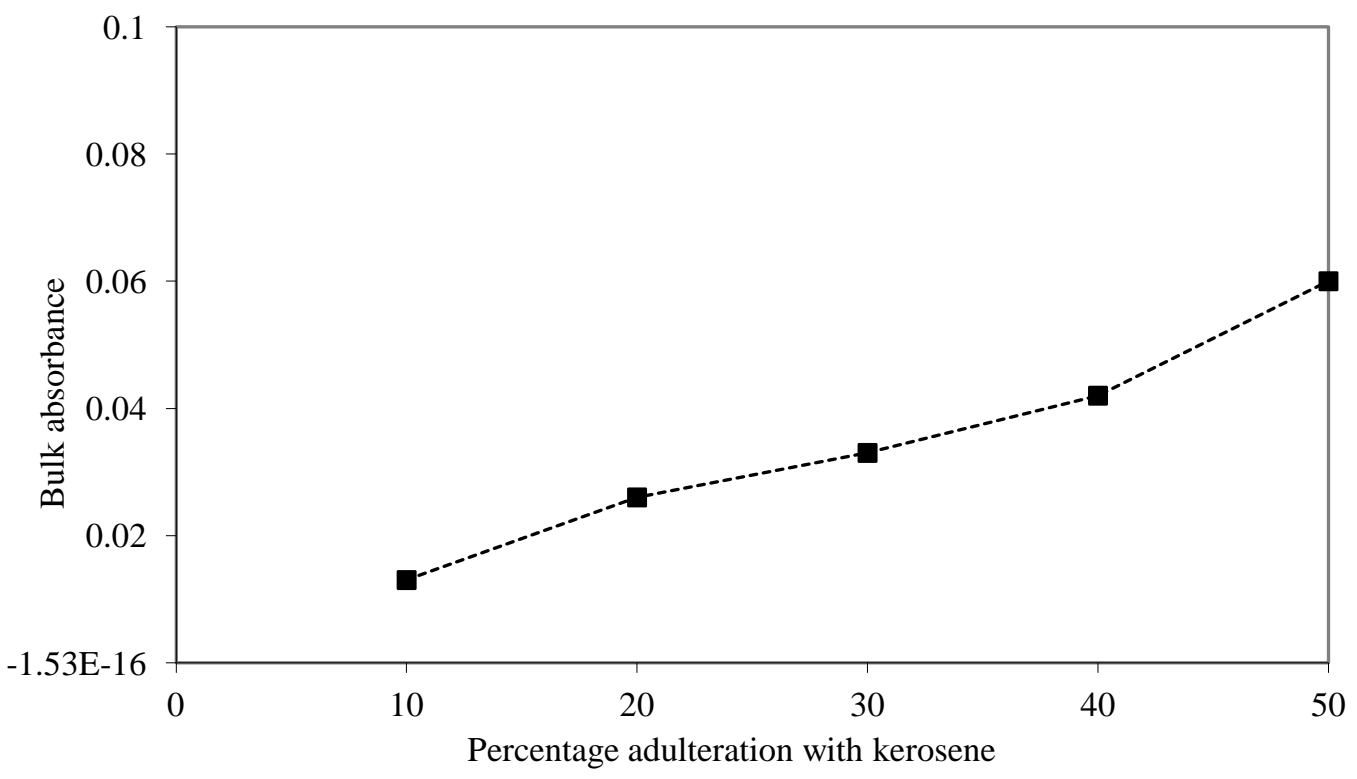

Figure 2. Variation of bulk absorbance of diesel with kerosene adulteration. 


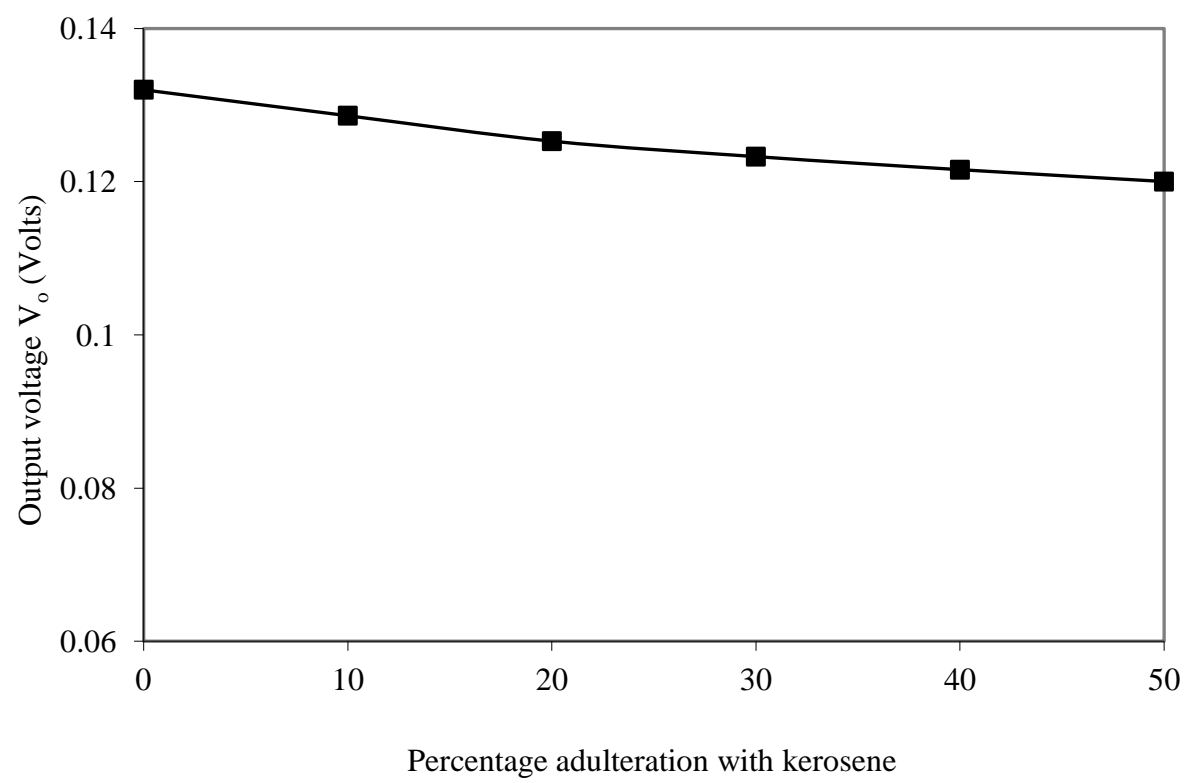

Figure 3. Output voltage for percentage adulteration with kerosene.

\section{CONCLUSION}

An electronic technique has been devised for the determination of adulteration in diesel by kerosene utilizing optical characteristics of light. The relation between output voltage versus percentage concentration was presented by drawing calibration graph and was found to be linear. The adulteration was also determined using conventional UV-VIS spectrophotometer and the result were similar with the advantage that the proposed method is efficient, simple, compact and portable. This method can be routinely used in automobile and petrochemical industries. Besides this, the method can be used as a monitoring technique for quality control of diesel.

\section{REFERENCES}

[1] Srivastava, A. Shukla, R. K., and Bali, L. M. 1997. Optical sensor for determining adulteration in petrol by kerosene. In Proceedings of the International Conference on Fiber Optics and Photonics, New Delhi, Tata McGraw Hill, vol. II, pp. 989

[2] S. Roy, "Fiber optic sensor for determining adulteration of petrol and diesel by kerosene," Sensors and Actuators B, vol.55, 1999, 212-216.

[3] R. A. morello-frosch, T. J. Woodruff, D. A. Axelrad and J. C. Caldwell, "Air toxic and health risks in California: The public health implications of outdoor concentrations," Risk Analysis, vol. 20(2), pp. 273-291, 2000 .
[4] A. A. Gupta, K. K. Swami, A. K. Misra, A. K. Bhatnagar and P. K. Mukhopadhyay, "Marker-R \& D; a new marker system for kerosene," Hydrocarbon Technology, vol. 15, pp. 137- 151, 1992.

[5] V. P. Bhatnagar, "An ultrasonic method to find liquid fuel adulteration," J. Acoust. Soc. India, vol. 9, pp. 1923, 1981.

[6] M. Shahru Bahari, W. J. Criddle, and J. D. R. Thomas, "Determination of the adulteration of petrol with kerosene using rapid phase titration procedure," Analyst, vol. 115, pp. 417-419, 1990.

[7] M. Shahru Bahari, W. J. Criddle, and J. D. R. Thomas, "Spectrophotometric end-point phase-titration determination of the adulteration of petrol with kerosene," Anal. Proc., vol. 28, pp. 14-16, 1991.

[8] M. Shahru Bahari, W. J. Criddle, and J. D. R. Thomas, "Fuel cell methodology for determining petrol adulteration with kerosene," Anal. Proc. vol. 29, pp. 30$31,1992$.

[9] Jose Miguel Lopez-Higuera, Handbook of optical Fiber sensing technology, John Wiley \& Sons Ltd, 2001.

[10] P. H. Paul, and G. Kychakoff, "fiber-optic evanescent field absorption sensor," Appl. Phys. Lett. vol. 51, pp.1214, 1987.

[11] V. Ruddy, B. D. Maccraith and J. A. Murphy, "Evanescent wave absorption spectroscopy using multimode fibers,” J. Appl. Phys. vol. 67, pp.6070-6074, 1990. 